\title{
Autoimmune Manifestations in Heterozygote Type I Complement 2 Deficiency: A Child Eventually Diagnosed With Systemic Lupus Erythematosus
}

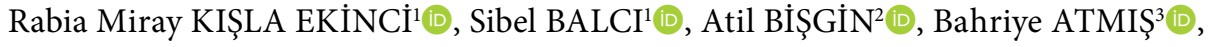 \\ Dilek DOĞRUEL ${ }^{4}$ iD, Mustafa YILMAZ ${ }^{1}$ (D) \\ ${ }^{1}$ Department of Pediatric Rheumatology, Medicine Faculty of Çukurova University, Adana, Turkey \\ ${ }^{2}$ Department of Medical Genetics, Medicine Faculty of Çukurova University, Adana, Turkey \\ ${ }^{3}$ Department of Pediatric Nephrology, Medicine Faculty of Çukurova University, Adana, Turkey \\ ${ }^{4}$ Department of Pediatic Allergy and Immunology, Medicine Faculty of Çukurova University, Adana, Turkey
}

\begin{abstract}
Systemic lupus erythematosus (SLE) is an autoimmune disorder resulting in a broad spectrum of manifestations in several organs, mainly skin and kidney. SLE occurs with interaction of genetic and environmental factors. The most remarkable genetic predisposition to SLE is deficiency of early components of the classical complement pathway. A five-year-old, previously healthy female patient was admitted to our hospital with headache, fever, focal partial seizure, diagnosed and treated as encephalitis. She was re-admitted to our hospital at six years of age with fever, fatigue, alopecia and oral aphthous ulcers and necrotizing vasculitis on extremities. Significant hypocomplementemia, anemia, proteinuria and positive autoantibodies and coombs test led to the diagnosis of SLE. Due to early disease onset and distinct autoimmune manifestations, we diagnosed our patient with type I complement 2 (C2) deficiency with a frameshift mutation in C2 gene and a serum C2 level of $<0.2$ mg/dL. To our knowledge, this is the first case of genetically confirmed and successfully treated hereditary C2 deficient SLE patient diagnosed with necrotizing vasculitis. We wish to highlight that distinctive autoimmune manifestations should guide physicians to research on monogenic lupus, particularly $C 2$ deficiency, even in the absence of coexisting recurrent pyogenic infections.

Keywords: Child, complement 2, cutaneous vasculitis, systemic lupus erythematosus.
\end{abstract}

Systemic lupus erythematosus (SLE) is an autoimmune disorder associated with interaction of genetic and environmental factors and a broad spectrum of manifestations in several organs, mainly skin and kidney. ${ }^{1}$ Childhood SLE represents $15-20 \%$ of all SLE patients and its course is more severe with higher incidence of nephritis and hematologic disorders. ${ }^{2}$

Complement system activation by presence of immune complexes is known to be the leading cause of inflammation in SLE. It is not surprising that the most remarkable genetic predispositions to SLE are deficiencies of early components of classical complement pathway. ${ }^{1}$ In this article, we report a pediatric patient who presented with autoimmune manifestations, met SLE criteria over time and was finally diagnosed with heterozygote type I complement 2 deficiency (C2D).

\section{CASE REPORT}

A five-year-old female patient was admitted to our hospital with headache and fever for the last five days. Brain magnetic resonance imaging

Received: February 23, 2018 Accepted: March 30, 2018 Published online: May 03, 2018

Correspondence: Rabia Miray Kisla Ekinci, MD. Çukurova Üniversitesi Tıp Fakültesi Çocuk Romatolojisi Bilim Dalı, 01330 Sarıçam, Adana, Turkey. Tel: +90 507 - 2171190 e-mail:mir_kisla@hotmail.com

Citation:

Kışla Ekinci RM, Balcı S, Bişgin A, Atmış B, Doğruel D, Yılmaz M. Autoimmune manifestations in heterozygote type I complement 2 deficiency: A child eventually diagnosed with systemic lupus erythematosus. Arch Rheumatol 2019;34(1):96-99. 

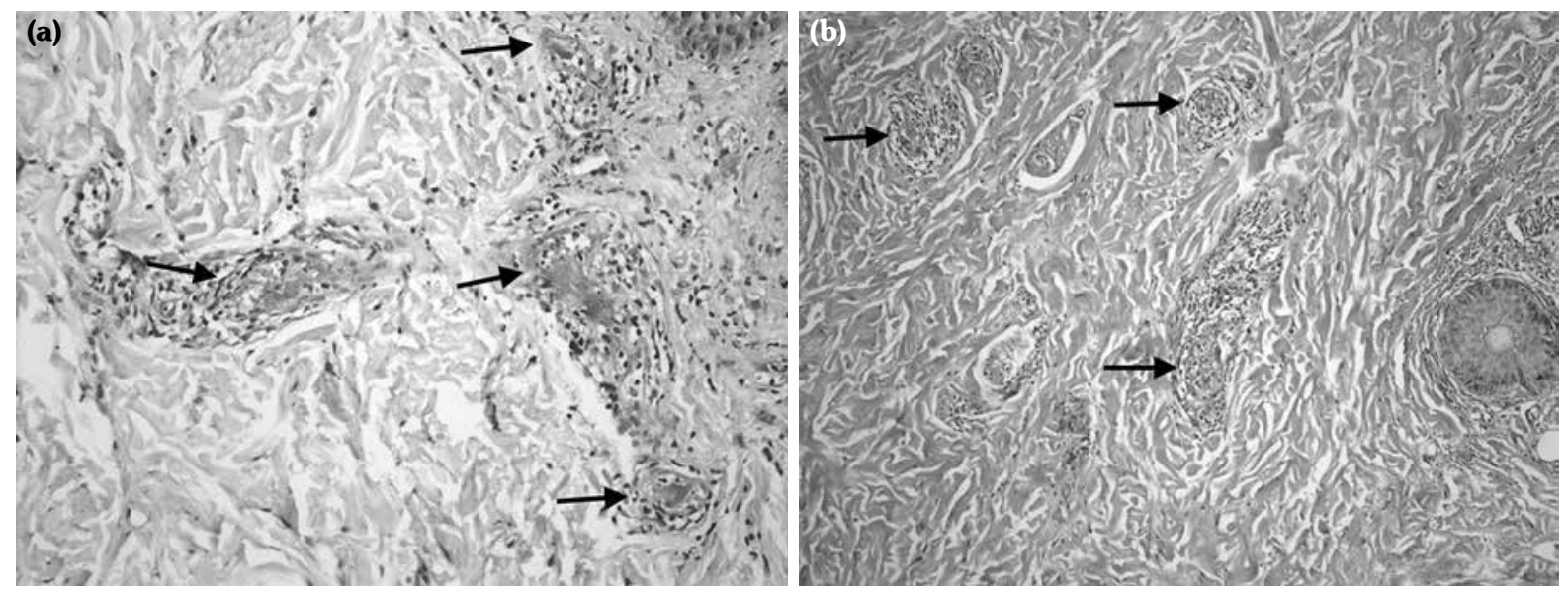

Figure 1. Pathological features of skin in our patient with systemic lupus erythematosus, revealing cutaneous necrotizing vasculitis. (a) Fibrinoid necrosis of vessel wall (periodic acid-Schiff stain $\times 200$ ). (b) Neutrophil and lymphocyte infiltration in vessel wall $(\mathrm{H}-\mathrm{E} \times 20)$.

showed bilateral focal lesions but particularly in right hippocampal gyrus and basal ganglions compatible with encephalitis. With partial benefit from treatment with vancomycin, meropenem and acyclovir, the patient had developed focal partial seizure lasting 10 minutes on the right side of her face and right hemiparesis on the $12^{\text {th }}$ day of hospitalization. Electroencephalogram showed epileptiform activity originating from left hemicortex and the patient was successfully treated with phenytoin, phenobarbital and carbamazepine subsequently. Neurological manifestations clinically improved and hemiparesis almost disappeared at second month after discharge.

The patient was re-admitted to our hospital at six years of age with fever, fatigue and alopecia. Physical examination revealed multiple oral aphthous ulcers and necrotic ulcers on the left arm and leg. In laboratory tests, acute phase reactants were markedly increased, significant hypocomplementemia, anemia and $0.4 \mathrm{~g} /$ day proteinuria were present. Anti-nuclear antibody (ANA) was positive at $1 / 100$ titer in immunofluorescence assay. Additionally, direct Coombs, anti-Smith antibody and anti-double stranded deoxyribonucleic acid were positive. Skin biopsy showed cutaneous necrotizing vasculitis (Figure 1). SLE was diagnosed according to American College of Rheumatology classification criteria for SLE. ${ }^{3}$ Renal biopsy was planned but delayed due to the anticoagulant medications and possible vasculitic process. In the studies for hypercoagulability, serum homocysteine was slightly elevated while antithrombin 3 was mildly reduced and protein $\mathrm{C}$ and free protein $S$ levels were markedly reduced. Anticardiolipin immunoglobulin $G$ was positive, while immunoglobulin $\mathrm{M}$ was negative. Furthermore, genetic analysis revealed homozygote MTHFR A1298C mutation. After five doses of intravenous pulse metilprednisolone, plasmapheresis and prophylaxis with low molecular weight heparin (LMWH), remission was maintained with six pulses of monthly intravenous cyclophosphamide, consequent mycophenolate sodium treatment and systemic steroids. LMWH was discontinued after three months; serum C4 and $\mathrm{C} 3$ returned to normal range within two months and remained normal at six years of follow-up. She suffered from mild upper respiratory tract infections only four times a year. Due to discrete autoimmune manifestations including encephalitis, necrotizing vasculitis and SLE, we investigated our patient for early complement deficiencies. With new generation sequencing system, we detected c.21702170delC (p.P724Rfs*16) frameshift mutation causing premature stop codon. Immunochemical quantitation of $\mathrm{C} 2$ antigen by radial immunodiffusion confirmed the presence of type I C2D with a serum C2 level of $<0.2 \mathrm{mg} / \mathrm{dL}$. Table 1 summarizes the laboratory results of the patient. A written informed consent was obtained from the parents. 
Table 1. Laboratory results of patient with $C 2$ deficiency and systemic lupus erythematosus

\begin{tabular}{|c|c|c|c|}
\hline Parameters & At diagnosis & At the $6^{\text {th }}$ month of treatment & 7 years after the diagnosis \\
\hline Hemoglobin (gr/dL) & 8.3 & 13.3 & 13.4 \\
\hline Leukocytes $\left(/ \mathrm{mm}^{3}\right)$ & 4,920 & 6,010 & 5,180 \\
\hline Neutrophil $\left(/ \mathrm{mm}^{3}\right)$ & 2,120 & 3,060 & 2,100 \\
\hline Lymphocytes $\left(/ \mathrm{mm}^{3}\right)$ & 2,340 & 1,682 & 2,600 \\
\hline Thrombocytes $\left(/ \mathrm{mm}^{3}\right)$ & 169,000 & 309,000 & 326,000 \\
\hline $\operatorname{ESR}(\mathrm{mm} / \mathrm{h})$ & 26 & 22 & 15 \\
\hline $\mathrm{CRP}(\mathrm{mg} / \mathrm{dL})$ & 1.06 & 2.5 & 0.4 \\
\hline $\mathrm{C} 3$ (mg/dL) & 33 & 110 & 112 \\
\hline $\mathrm{C} 4$ (mg/dL) & 7.1 & 22 & 25.1 \\
\hline ANA (titer, IFA) & $1 / 100$ & Not-determined & Not-determined \\
\hline ANA (U/mL, ELISA) & 54.9 & 75.1 & Negative \\
\hline Anti-DNA (U/mL, ELISA) & 20 & Negative & 55 \\
\hline Anti-Sm (U/mL, ELISA) & 194.8 & Not-determined & 10.4 \\
\hline Urine protein ( $\mathrm{g} / \mathrm{d}$ urine) & 0.4 & 0.2 & Not-determined \\
\hline Direct coombs & Positive (+3) & Not-determined & Negative \\
\hline $\mathrm{LDH}(\mathrm{mg} / \mathrm{dL})$ & 673 & 199 & 175 \\
\hline Anticardiolipin IgG & Positive & Positive & Negative \\
\hline Anticardiolipin IgM & Negative & Negative & Negative \\
\hline $\begin{array}{l}\text { Anti- } \beta 2 \text { glycoprotein I } \\
\text { antibodies }\end{array}$ & Negative & Negative & Negative \\
\hline Protein C (70-130\%) & 52 & 21 & 70.2 \\
\hline Free protein S (60-125 \%) & 51 & 16.9 & 100.6 \\
\hline Antithrombin 3 (79-125\%) & 74 & 118.4 & 72.4 \\
\hline
\end{tabular}

\section{DISCUSSION}

Systemic lupus erythematosus is a multi-systemic autoimmune disease demonstrating complement consumption and type I interferon signaling. There is also an imbalance in apoptosis and apoptotic material removal, which results in break in tolerance and antibody production against self-antigens. In recent years, description of monogenic lupus-like disorders has improved our understanding of SLE. ${ }^{1}$

Deficiencies of early components of complement system were the first defined monogenic causes of lupus. Autoimmunity may occur due to altered opsonization, immune complex removal from the circulation, clearance of apoptotic debris, and lack of normal $\mathrm{B}$ cell tolerance, overwhelming production of autoantibodies in C2D. ${ }^{4}$

Systemic lupus erythematosus or lupuslike manifestations prevalence among early complement deficiencies is reported in $10 \%$ of C2D. Severe recurrent infections associated with high mortality may be occasionally present in homozygote complement deficiencies. ${ }^{5}$ On the other hand, our patient had only suffered from recurrent mild pharyngitis.

While the frequency of heterozygous $\mathrm{C} 2 \mathrm{D}$ is $1-2 \%$ in Caucasian populations, its prevalence 
is higher in SLE patients with 2.4-5.8\%.1,5 C2 deficient SLE patients typically present with renal disease, arthritis, malar rash, discoid rash and photosensitivity. ${ }^{1,6}$ Our patient clinically differed from the patients in the literature with the presence of oral aphthous, cutaneous vasculitis, hematological and neurological findings and the absence of arthritis or other skin findings.

Immunological analysis of C2D patients with SLE has shown low titer positive ANA and a distinctive profile of negative antideoxyribonucleic acid and positive anti-Sjögren's syndrome A and anti-ribonucleoprotein. Despite the absence of anti-phospholipid syndrome (APLS), patients with C2D tended to have positive anti-cardiolipin antibodies. ${ }^{5,6}$ Similarly, our patient had transiently positive anticardiolipin antibody without clinical APLS. She was transiently positive for ANA and anti-Smith antibodies with high titers, in contrast to the literature. Although our patient did not have any thrombotic event, she had multiple hereditary and acquired factors for hypercoagulation such as homozygote MTHFR A1298C mutation, transiently reduced levels of protein $\mathrm{C}$ and $\mathrm{S}$. To our knowledge, these additional factors do not seem to increase thrombotic risk in SLE patients. ${ }^{7}$

Regarding genotype; type I C2D, usually caused by a deletion and generation of a stop codon in $\mathrm{C} 2$ gene, results in the absence of detectable serum C2. Type II C2D leads to reduced plasma levels of $\mathrm{C} 2$ caused by particularly a point mutation. ${ }^{1}$ Our case was diagnosed as SLE and type I C2D with a novel c.2170-2170 delC (p.P724Rfs*16) frameshift mutation causing premature stop codon and undetectable serum levels of serum C2.

Since necrotic skin ulcers and vasculitis are unusual in SLE course and C2D, the most distinctive finding was cutaneous necrotizing vasculitis in our patient. 8,9 To the best of our knowledge, this is the first case of genetically confirmed and successfully treated hereditary C2 deficient SLE patient diagnosed with necrotizing vasculitis. We wish to highlight that early disease onset or distinctive autoimmune manifestations should guide physicians to research on monogenic lupus, particularly $\mathrm{C} 2 \mathrm{D}$, even in the absence of coexisting recurrent pyogenic infections.

\section{Declaration of conflicting interests}

The authors declared no conflicts of interest with respect to the authorship and/or publication of this article.

\section{Funding}

The authors received no financial support for the research and/or authorship of this article.

\section{REFERENCES}

1. Macedo AC, Isaac L. Systemic lupus erythematosus and deficiencies of early components of the complement classical pathway. Front Immunol 2016;7:55.

2. Papadimitraki ED, Isenberg DA. Childhood- and adultonset lupus: an update of similarities and differences. Expert Rev Clin Immunol 2009;5:391-403.

3. Hochberg MC. Updating the American College of Rheumatology revised criteria for the classification of systemic lupus erythematosus. Arthritis Rheum 1997;40:1725.

4. Truedsson L, Bengtsson AA, Sturfelt G. Complement deficiencies and systemic lupus erythematosus. Autoimmunity 2007;40:560-6.

5. Kallel-Sellami M, Laadhar L, Zerzeri Y, Makni S. Complement deficiency and systemic lupus erythematosus: consensus and dilemma. Expert Rev Clin Immunol 2008;4:629-37.

6. Lipsker DM, Schreckenberg-Gilliot C, UringLambert B, Meyer A, Hartmann D, Grosshans EM, et al. Lupus erythematosus associated with genetically determined deficiency of the second component of the complement. Arch Dermatol 2000;136:1508-14.

7. Sallai KK, Nagy E, Bodó I, Mohl A, Gergely P. Thrombosis risk in systemic lupus erythematosus: the role of thrombophilic risk factors. Scand J Rheumatol 2007;36:198-205.

8. Gheita TA, Abaza NM, Sayed S, El-Azkalany GS, Fishawy HS, Eissa AH. Cutaneous vasculitis in systemic lupus erythematosus patients: potential key players and implications. Lupus 2017:961203317739134.

9. Bennett RE, Clough JD, Calabrese LH. Fulminating vasculitis in a homozygous C2-deficient patient. Cleve Clin Q 1985;52:213-5. 